\title{
Regional stability and stabilization of a class of linear hyperbolic systems with nonlinear quadratic dynamic boundary conditions
}

\author{
André F. Caldeira ${ }^{1 \mathrm{a}}$, Christophe Prieur ${ }^{\mathrm{b}}$, Daniel Coutinho ${ }^{\mathrm{c}}$, Valter J. S. \\ Leite $^{\mathrm{d}}$ \\ ${ }^{a}$ Graduate Program on Automation and Systems Engineering, UFSC, PO BOX 476, \\ 88040 - 900, Florianópolis, SC, Brazil, and Grenoble Image Parole Signal Automatique \\ (GIPSA-lab), F-38000 Grenoble, France. caldeiraaf@gmail.com \\ ${ }^{b}$ Univ. Grenoble Alpes, CNRS, Grenoble INP, GIPSA-lab, F-38000 Grenoble, France. \\ christophe.prieur@gipsa-lab.fr \\ ${ }^{c}$ Department of Automation and Systems, UFSC, PO BOX 476, 88040 - 900, \\ Florianópolis, $S C$, Brazil. coutinho@das.ufsc.br \\ ${ }^{d}$ Department of Mechatronics Engineering, CEFET-MG-Campus Divinópolis, $R$. \\ Álvares Azevedo, 400, 35503-822, Divinópolis, MG, Brazil. valter@ieee.org
}

\begin{abstract}
This paper addresses the boundary control problem of fluid transport in a Poiseuille flow taking the actuator dynamics into account. More precisely, sufficient stability conditions are derived to guarantee the exponential stability of a linear hyperbolic differential equation system subject to nonlinear quadratic dynamic boundary conditions by means of Lyapunov based techniques. Then, convex optimization problems in terms of linear matrix inequality constraints are derived to either estimate the closed-loop stability region or synthesize a robust control law ensuring the local closed-loop stability while estimating an admissible set of initial states. The proposed results are then applied to application-oriented examples to illustrate local stability and stabilization tools.
\end{abstract}

Keywords: dynamic boundary conditions, Poiseuille flow, linear hyperbolic systems, robust control, LMIs.

\footnotetext{
${ }^{1}$ A. F. Caldeira was pursuing a doctoral degree in the Graduate Program on Automation and Systems Engineering, UFSC, and École Doctorale Electronique, Electrotechnique, Automatique, Traitement du Signal, Univ. Grenoble Alpes.
} 


\section{Introduction}

In industrial processes, a large variety of physical systems is governed by hyperbolic partial differential equations (PDEs) such as hydraulic networks $[4,21]$ and gas devices $[11,12]$. In particular, fluid transport is often modeled by balance laws (or conservation laws when additive and dissipative terms are disregarded) which are hyperbolic PDEs normally used to express the fundamental dynamics of open conservative systems. However, infinite dimensional systems introduce variable time-delays making the closed-loop control much more challenging and, moreover, distributed measurements and actuators are not usually available. As a consequence, it is more common that actuators and measurements are located at the boundaries which is desired in practical applications as, for instance, in the references previously cited. In addition, a large number of numerical techniques based on finite-dimensional tools, which are often used to the stability analysis of PDE systems, provides only approximate solutions.

On the other hand, Lyapunov theory has been largely applied for several decades to deal with the stability analysis and control design of finite dimensional systems described by ordinary differential equations (ODEs) [37]. In the particular case of linear dynamical systems, a large number of stability and stabilization results are cast in terms of linear matrix inequality (LMI) constraints [6], which are numerically solved using dedicated software [30]. The LMI framework is a powerful tool for linear and nonlinear finitedimensional systems, since it can deal with a large diversity of control and systems theory problems such as robust stability, domain of attraction estimation, input-to-output performance, state or dynamic output-feedback control, and state estimation (see [6, 7, 32, 18, 13, 24] among other references). As a result, LMIs have been successfully applied in a wide diversity of control oriented applications as, for instance, wind turbine operation, satellite attitude regulation, turbo-charged combustion engine control and bioprocess control and estimation $[31,19]$.

In the context of infinite dimensional systems, the stability problem of boundary control for first-order hyperbolic systems using quadratic strict Lyapunov functions was more recently stated; see, for instance, [16, 15, 33]. A common assumption in most of available results is that the boundary action is faster than the wave travel making possible to establish a static relationship between the control input and the boundary condition. However, there are applications where the dynamics associated to the boundary control action 
cannot be neglected as, for instance, in the temperature control of an airflow in a heating column. To deal with dynamic boundary actions, the infinitedimensional system discretization together with the use of finite-dimensional tools are often employed. On the other hand, several approaches considering infinite-dimensional based techniques have been recently proposed. To cite a few, some sufficient conditions for the exponential stability of hyperbolic systems with linear time invariant dynamic boundary conditions were proposed in [10], the backstepping technique for the boundary control of hyperbolic and time-delayed systems was considered in [28], the dynamic boundary stabilization of linear parameter-varying (LPV) hyperbolic systems using the LMI framework was studied in [12], the problem of stability analysis and control synthesis for first-order hyperbolic linear PDEs over a bounded interval with spatially varying coefficients was the focus of [29], and a multimodel approach with a bilinear matrix inequality to deal with the stability of a hyperbolic system representing the flow in a open channel was proposed in [23]. It turns out that the majority of the latter references consider only dynamic boundary actions described by linear differential equations.

This paper addresses the boundary stabilization of uncertain first-order hyperbolic systems with boundary actions governed by an uncertain nonlinear quadratic differential equation. It should be emphasized that the class of quadratic systems can describe state-space models containing quadratic nonlinearities in the state variables and bilinear terms involving the state and control signal. Moreover, this class of systems can represent a large number of processes such as distillation and heating columns [25, 10], induction motors [3] and DC-DC converters [35], which has attracted recurring interest of the control practitioners; see, for instance, $[2,1,36,17]$ and references therein. Notice, in the context of infinite dimensional systems, that boundary actions are often nonlinear as, for instance, in the geometric average particle volume regulation of plug-low reactors, flow regulation in open channels delimited by overflow spillways and the vibration rejection in flexible marine risers $[14,16,26]$. In particular, local boundary stabilization conditions for the coupled linear first-order hyperbolic system and nonlinear quadratic dynamic boundary actuation are in this paper proposed in terms of a finite set of LMI constraints based on the Lyapunov stability theory. The local stability is characterized in a regional context, that is, bounded initial states will imply that the state trajectories remain bounded and they converge to the equilibrium point as the time goes to infinity. Hence, an optimization problem is proposed for the boundary feedback control design in order to de- 
termine a quadratic boundary state feedback control law that maximizes the set of admissible boundary initial conditions while guaranteeing the stability of the coupled ODE-PDE system.

The remaining of this paper is organized as follows. The problem of interest is stated in Section 2. Then, Section 3 presents the main stability results for uncertain first-order linear hyperbolic system subject to nonlinear boundary conditions. Next, the stability results are adapted in Section 4 to control synthesis by means of an appropriate similarity transformation where a stabilizing nonlinear boundary state feedback control law is designed in order to maximize the region of admissible initial conditions. Then, Section 5 provides two application-based examples in order to demonstrated the potentials of the proposed approach. Finally, Section 6 ends the paper giving some concluding remarks.

Notation: $\mathbb{R}^{+}=[0,+\infty), \mathbb{R}^{n}$ is the $n$-dimensional Euclidean space, $\mathbb{R}^{m \times n}$ is the set of $m \times n$ real matrices with real entries, $\mathbf{I}_{n}$ in the $n \times n$ identity matrix, $\mathbf{0}_{n}$ is the $n \times n$ zeros matrix and $\operatorname{diag}\{\cdots\}$ denotes a block-diagonal matrix. For a real matrix $S, S^{\prime}$ denotes its transpose, $\operatorname{He}\{S\}$ stands for $S+S^{\prime}$, and $S>0$ means that $S$ is symmetric and positive-definite. For two polytopes $\mathcal{X}$ and $\mathcal{Y}, \mathcal{X} \times \mathcal{Y}$ denotes the meta-polytope obtained by cartesian product and $\mathcal{V}(\mathcal{X})$ is the set of all vertices of $\mathcal{X}$. For a vector $\xi \in \mathbb{R}^{n}$, the $k$-component of $\xi$ is denoted by $\xi_{(k)}$. The usual Euclidian norm in $\mathbb{R}^{n}$ is denoted by $\|\cdot\|$, whereas the set of all functions $\phi:(0, L) \rightarrow \mathbb{R}^{n}$ such that

$\int_{0}^{L} \phi(x)^{\prime} \phi(x) d x<\infty$ is denoted by $L^{2}\left((0, L) ; \mathbb{R}^{n}\right)$ that is equipped with the norm $\|\cdot\|_{L^{2}\left((0, L) ; \mathbb{R}^{n}\right)}$. Given a topological set $\mathcal{J}$, and an interval $\mathcal{I}$ in $\mathbb{R}^{+}$, the set $\mathcal{C}^{0}(\mathcal{I}, \mathcal{J})$ is the set of continuous functions $\phi: \mathcal{I} \rightarrow \mathcal{J}$.

\section{Problem Statement}

Let $n$ be a positive integer, $\Omega$ an open non-empty set of $\mathbb{R}^{n}$ and $\Delta$ a non empty convex set of $\mathbb{R}^{n_{\delta}}$. Consider the general class of first-order uncertain hyperbolic systems of order $n$ defined as follows:

$$
\partial_{t} \boldsymbol{\xi}(t, x)+\boldsymbol{\Lambda}(\delta) \partial_{x} \boldsymbol{\xi}(t, x)=\mathbf{0}, \quad t \in \mathbb{R}^{+}, \quad x \in[0, L],
$$

where $\boldsymbol{\xi}: \mathbb{R}^{+} \times[0, L] \rightarrow \Omega, \delta \in \Delta$ is a bounded sufficiently smooth vector function of time; $\partial_{t}$ and $\partial_{x}$ represent (first order) partial derivatives with respect to time and space, respectively; $\Lambda(\delta): \Delta \rightarrow \mathbb{R}^{n \times n}$ is a diagonal and continuous matrix function (called the characteristic matrix), i.e., $\boldsymbol{\Lambda}(\delta)=$ 
$\operatorname{diag}\left\{\lambda_{1}(\delta), \lambda_{2}(\delta), \ldots, \lambda_{n}(\delta)\right\}$ with $\lambda_{i}(\delta)$ being positive continuous functions, for all $\delta \in \Delta$ and $i=1, \ldots, n$.

Assumption 1. The diagonal elements of $\boldsymbol{\Lambda}(\delta) \in \mathbb{R}^{n \times n}$ satisfy the following inequalities for all $\delta \in \Delta$ :

$$
0<\lambda_{1}(\delta)<\cdots<\lambda_{n}(\delta)
$$

Remark 1. Assumption 1 implies that system (1) has only positive convecting speeds, however it does not add any loss of generality. Note that, for first order hyperbolic systems with both negative and positive convecting speeds, there always exists a variable transformation to obtain a system as in (1) satisfying Assumption 1 (see the beginning of proof of Theorem 1 in [10], or Page 7 of [34] or Remark 1 of [12] or Remark 3 of [11]). In particular, consider system (1) with the diagonal elements of $\boldsymbol{\Lambda}(\delta)$ ordered as follows

$$
\lambda_{1}(\delta)<\cdots<\lambda_{m}(\delta)<0<\lambda_{m+1}(\delta)<\cdots<\lambda_{n}(\delta), \forall \delta \in \Delta,
$$

and let $\boldsymbol{\xi}: \mathbb{R}^{+} \times[0, L] \rightarrow \Omega \in \mathbb{R}^{n}$ be partitioned as

$$
\boldsymbol{\xi}(t, x)=\left[\begin{array}{l}
\boldsymbol{\xi}_{-}(t, x) \\
\boldsymbol{\xi}_{+}(t, x)
\end{array}\right], \boldsymbol{\xi}_{-} \in \mathbb{R}^{m}, \boldsymbol{\xi}_{+} \in \mathbb{R}^{n-m} .
$$

Then, applying the transformation

$$
\tilde{\boldsymbol{\xi}}(t, x)=\left[\begin{array}{c}
\boldsymbol{\xi}_{-}(t, L-x) \\
\boldsymbol{\xi}_{+}(t, x)
\end{array}\right]
$$

yields a system as in (1) with only positive convection speeds.

Associated with (1), consider the following nonlinear quadratic dynamic boundary action:

$$
\left\{\begin{aligned}
\dot{\boldsymbol{\xi}}_{\text {in }}(t) & =A\left(\boldsymbol{\xi}_{\text {in }}(t), \delta\right) \boldsymbol{\xi}_{\text {in }}(t)+B\left(\boldsymbol{\xi}_{\text {in }}(t), \delta\right) u(t), \\
u(t) & =G\left(\boldsymbol{\xi}_{\text {in }}(t)\right) \boldsymbol{\xi}_{\text {in }}(t)+K \boldsymbol{\xi}_{\text {out }}(t),
\end{aligned}\right.
$$

where

$$
\boldsymbol{\xi}_{\text {in }}(t)=\boldsymbol{\xi}(t, 0), \boldsymbol{\xi}_{\text {out }}(t)=\boldsymbol{\xi}(t, L)
$$

are the boundary conditions of (1) interconnecting the boundary dynamics of (1) with (2), $\boldsymbol{\xi}_{\text {in }} \in \Xi \subset \mathbb{R}^{n}$ and $\boldsymbol{\xi}_{\text {out }} \in \mathbb{R}^{n}$ are the state vectors of the dynamic 
boundary condition at $x=0$ and $x=L$, respectively, and assumed to be measurable, $u(t) \in \mathbb{R}^{n_{u}}$ is the boundary control input, $\Xi$ is compact region of the boundary state-space containing $\boldsymbol{\xi}_{\text {in }}=0$ and to be specified later in this paper. Notice to ease the presentation that the system and boundary dynamics uncertainties are merged into the vector $\delta$, i.e., the elements of $\delta$ comprise both system and boundary dynamics modeling errors.

Further, $K \in \mathbb{R}^{n_{u} \times n}$ is a real constant matrix and $A(\cdot) \in \mathbb{R}^{n \times n}, B(\cdot) \in$ $\mathbb{R}^{n \times n_{u}}, G(\cdot) \in \mathbb{R}^{n_{u} \times n}$ are affine matrix functions on their arguments, that is:

$$
\begin{aligned}
& {\left[A\left(\boldsymbol{\xi}_{i n}, \delta\right) \quad B\left(\boldsymbol{\xi}_{i n}, \delta\right)\right]=\left[\begin{array}{ll}
A_{0} & B_{0}
\end{array}\right]+\sum_{i=1}^{n} \boldsymbol{\xi}_{i n(i)}\left[\begin{array}{ll}
A_{i} & B_{i}
\end{array}\right]} \\
& +\sum_{j=1}^{n_{\delta}} \delta_{(j)}\left[\begin{array}{cc}
\breve{A}_{j} & \breve{B}_{j}
\end{array}\right] \\
& G\left(\boldsymbol{\xi}_{\text {in }}\right)=G_{0}+\sum_{i=1}^{n} \boldsymbol{\xi}_{\text {in }(i)} G_{i},
\end{aligned}
$$

with $A_{i}, B_{i}$, and $G_{i}, i=0,1, \ldots, n$, and $\breve{A}_{j}, \breve{B}_{j}, j=1, \ldots, n_{\delta}$, being given constant real matrices with appropriate dimensions. It is assumed that the pair $\left(A_{0}, B_{0}\right)$ is stabilizable and the unforced system of $(2)$ is allowed to be unstable.

In addition, the initial conditions of the coupled PDE-ODE system of (1) and (2), namely

$$
\boldsymbol{\xi}(0, x)=\overline{\boldsymbol{\xi}}(x) \quad \text { and } \quad \boldsymbol{\xi}_{\text {in }}(0)=\overline{\boldsymbol{\xi}}_{\text {in }},
$$

are assumed to satisfy:

$$
\overline{\boldsymbol{\xi}} \in \mathcal{D}_{1} \quad \text { and } \quad \overline{\boldsymbol{\xi}}_{\text {in }} \in \mathcal{D}_{2}
$$

where the sets $\mathcal{D}_{1}$ and $\mathcal{D}_{2}$ are defined as follows

$$
\begin{aligned}
& \mathcal{D}_{1}:=\left\{\overline{\boldsymbol{\xi}} \in L^{2}\left((0, L) ; \mathbb{R}^{n}\right): \int_{0}^{L} \overline{\boldsymbol{\xi}}(x)^{\prime} \overline{\boldsymbol{\xi}}(x) d x \leq \sigma\right\}, \\
& \mathcal{D}_{2}:=\left\{\overline{\boldsymbol{\xi}}_{\text {in }} \in \mathbb{R}^{n}: \overline{\boldsymbol{\xi}}_{\text {in }}{ }^{\prime} P_{1} \overline{\boldsymbol{\xi}}_{\text {in }} \leq 1\right\}
\end{aligned}
$$

with the scalar $\sigma>0$ and the matrix $P_{1}>0$ defining the sizes of $\mathcal{D}_{1}$ and $\mathcal{D}_{2}$, respectively. In the particular case of linear dynamic boundary conditions, 
that is, when $A_{i}, B_{i}, \check{A}_{i}$ and $\check{B}_{i}$ are null matrices, for $i=1, \ldots, n$, the existence and uniqueness of solutions to (2), (3) and (6) for initial conditions satisfying (7) is ensured, see e.g. [15]. As these solutions may not be differentiable everywhere, the concept of weak solutions to partial differential equations has to be used; see [21] and references therein for further details.

In view of the above scenario, this paper is concerned in obtaining sufficient conditions to the regional stability and stabilization problems for coupled PDE-ODE system of (1) and (2) as stated below:

$\boldsymbol{P} \mathbf{1}$ For given state feedback gain matrices $G\left(\boldsymbol{\xi}_{\text {in }}\right)$ and $K\left(\boldsymbol{\xi}_{\text {out }}\right)$ as defined in (5), derive stability analysis conditions ensuring the robust local stability of the coupled PDE-ODE system while determining a maximized set of initial boundary states $\mathcal{D}_{2}$ for a given set $\mathcal{D}_{1}$.

$\boldsymbol{P 2}$ Design the state feedback gain matrices $G\left(\boldsymbol{\xi}_{\text {in }}\right)$ and $K\left(\boldsymbol{\xi}_{\text {out }}\right)$ as defined in (5), ensuring the robust local stability of the coupled PDE-ODE system while maximizing the set of initial boundary states $\mathcal{D}_{2}$ for a given set $\mathcal{D}_{1}$.

Before ending this section, the notion of exponential stability to be considered in this paper is introduced for the coupled PDE-ODE system of (1) and (2).

Definition 1. The coupled PDE-ODE system of (1) and (2), with initial conditions $\overline{\boldsymbol{\xi}}$ and $\overline{\boldsymbol{\xi}}_{\text {in }}$ satisfying (7), is said to be locally robustly exponentially stable if there exist positive scalars $\alpha$ and $\beta$ such that the following holds:

$$
\begin{aligned}
& \left(\left\|\boldsymbol{\xi}_{\text {in }}(t)\right\|+\|\boldsymbol{\xi}(t, \cdot)\|_{L^{2}\left((0, L) ; \mathbb{R}^{n}\right)}\right) \leq \beta \mathrm{e}^{-\alpha t}\left(\left\|\overline{\boldsymbol{\xi}}_{\text {in }}\right\|+\|\overline{\boldsymbol{\xi}}\|_{L^{2}\left((0, L) ; \mathbb{R}^{n}\right)}\right), \\
& \forall t \in \mathbb{R}^{+}, \delta \in \Delta .
\end{aligned}
$$

\section{Local Stability Analysis}

In this section, it is developed an LMI approach to derive a numerical and tractable solution to the robust regional stability analysis problem $\boldsymbol{P} \mathbf{1}$ as defined in Section 2. To this end, consider the following Lyapunov function candidate defined for all continuously differentiable functions $\boldsymbol{\xi}: \mathbb{R}^{+} \times[0, L] \rightarrow \Omega$ :

$$
V(\boldsymbol{\xi})=\boldsymbol{\xi}_{i n}^{\prime} P_{1} \boldsymbol{\xi}_{i n}+\int_{0}^{L} \mathrm{e}^{-\mu x} \boldsymbol{\xi}(x)^{\prime} P_{2} \boldsymbol{\xi}(x) d x
$$


where $P_{1}, P_{2} \in \mathbb{R}^{n \times n}$ are positive definite diagonal matrices and $\mu$ is a positive scalar.

Then, evaluating the time derivative of $V(\cdot)$ along the solutions to (1) and (2) with (7) leads to (see, for instance, [16] and [11] for further details):

$$
\begin{aligned}
\dot{V}(\boldsymbol{\xi}(t, \cdot))=2 \boldsymbol{\xi}_{\text {in }}(t)^{\prime} P_{1}\left(A\left(\boldsymbol{\xi}_{\text {in }}, \delta\right)+B\left(\boldsymbol{\xi}_{\text {in }}, \delta\right) G\left(\boldsymbol{\xi}_{\text {in }}\right)\right) \boldsymbol{\xi}_{\text {in }}(t) \\
+2 \boldsymbol{\xi}_{\text {in }}(t)^{\prime} P_{1} B\left(\boldsymbol{\xi}_{\text {in }}, \delta\right) K \boldsymbol{\xi}_{\text {out }}(t)-\left[\mathrm{e}^{-\mu x} \boldsymbol{\xi}(t, x)^{\prime} \boldsymbol{\Lambda}(\delta) P_{2} \boldsymbol{\xi}(t, x)\right]_{x=0}^{x=L} \\
-\mu \int_{0}^{L} \mathrm{e}^{-\mu x} \boldsymbol{\xi}(t, x)^{\prime} \boldsymbol{\Lambda}(\delta) P_{2} \boldsymbol{\xi}(t, x) d x
\end{aligned}
$$

In light of the above and taking (3) into account, $\dot{V}(\boldsymbol{\xi}(t, \cdot))$ can be cast as follows:

$$
\dot{V}(\boldsymbol{\xi}(t, \cdot))=-m_{1}(\boldsymbol{\xi}, \delta)+m_{2}\left(\boldsymbol{\xi}_{\text {in }}, \boldsymbol{\xi}_{\text {out }}, \delta\right)
$$

where

$$
\begin{aligned}
& m_{1}(\boldsymbol{\xi}, \delta)=\mu\left(\boldsymbol{\xi}_{\text {in }}^{\prime} \boldsymbol{\Lambda}(\delta) P_{1} \boldsymbol{\xi}_{\text {in }}+\int_{0}^{L} \mathrm{e}^{-\mu x} \boldsymbol{\xi}(t, x)^{\prime} \boldsymbol{\Lambda}(\delta) P_{2} \boldsymbol{\xi}(t, x) d x\right), \\
& m_{2}\left(\boldsymbol{\xi}_{\text {in }}, \boldsymbol{\xi}_{\text {out }}, \delta\right)=\boldsymbol{\xi}_{a}^{\prime} \Phi\left(\boldsymbol{\xi}_{\text {in }}, \delta\right) \boldsymbol{\xi}_{a}, \boldsymbol{\xi}_{a}=\left[\begin{array}{c}
\boldsymbol{\xi}_{\text {in }} \\
\boldsymbol{\xi}_{\text {out }}
\end{array}\right] \\
& \Phi\left(\boldsymbol{\xi}_{\text {in }}, \delta\right)=\left[\begin{array}{c|c}
A\left(\boldsymbol{\xi}_{\text {in }}, \delta\right)^{\prime} P_{1}+P_{1} A\left(\boldsymbol{\xi}_{\text {in }}, \delta\right) & \\
+G\left(\boldsymbol{\xi}_{\text {in }}\right)^{\prime} B\left(\boldsymbol{\xi}_{\text {in }}, \delta\right)^{\prime} P_{1} & P_{1} B\left(\boldsymbol{\xi}_{\text {in }}, \delta\right) K \\
+P_{1} B\left(\boldsymbol{\xi}_{\text {in }}, \delta\right) G\left(\xi_{\text {in }}\right) & \\
+\boldsymbol{\Lambda}(\delta) P_{2}+\mu \boldsymbol{\Lambda}(\delta) P_{1} & \\
\hline K^{\prime} B\left(\boldsymbol{\xi}_{\text {in }}, \delta\right)^{\prime} P_{1} & -\mathrm{e}^{-\mu} \boldsymbol{\Lambda}(\delta) P_{2}
\end{array}\right]
\end{aligned}
$$

Due to Assumption 1 and since $\boldsymbol{\Lambda}(\cdot)$ is a continuous function on the compact set $\Delta$, there exists a sufficiently small positive scalar $\epsilon$ such that $\epsilon \leq \lambda_{1}(\delta)$, for all $\delta \in \Delta$, which implies that $\epsilon \boldsymbol{I}_{n} \leq \boldsymbol{\Lambda}(\delta)$ for all admissible $\delta$.

Then, provided that

$$
m_{2}\left(\boldsymbol{\xi}_{\text {in }}, \boldsymbol{\xi}_{\text {out }}, \delta\right) \leq 0, \quad \forall\left(\boldsymbol{\xi}_{\text {in }}, \delta\right) \in \Xi \times \Delta, \boldsymbol{\xi}_{\text {out }} \in \mathbb{R}^{n}
$$

for a set $\Xi$ (that will be defined latter), the following holds

$$
\dot{V}(\boldsymbol{\xi}(t, \cdot)) \leq-\mu \epsilon V(\boldsymbol{\xi}(t)), \forall t \in \mathbb{R}^{+},
$$

from the fact that $m_{1}(\boldsymbol{\xi}(t, \cdot), \delta) \geq \mu \epsilon V(\boldsymbol{\xi}(t, \cdot))$. 
Notice that the inequality (13) implies that the coupled PDE-ODE system is locally exponentially stable if the condition (12) is satisfied along the solutions to (1) and (2) for all $\overline{\boldsymbol{\xi}} \in \mathcal{D}_{1}$ and $\overline{\boldsymbol{\xi}}_{\text {in }} \in \mathcal{D}_{2}$. Hence, we have to guarantee that the solution to the coupled PDE-ODE system is confined to a region

$$
\mathcal{R}(\gamma):=\{\boldsymbol{\xi}: V(\boldsymbol{\xi}) \leq \gamma\}
$$

for a certain $\gamma>0$.

Firstly, from (13), the following holds:

$$
V(\boldsymbol{\xi}(t, \cdot)) \leq \mathrm{e}^{-\mu \epsilon t} V(\overline{\boldsymbol{\xi}}), \forall \delta \in \Delta .
$$

Next, provided that $\overline{\boldsymbol{\xi}} \in \mathcal{D}_{1}, \overline{\boldsymbol{\xi}}_{\text {in }} \in \mathcal{D}_{2}$ and $\mu>0$, there exists a sufficiently large positive scalar $\varrho$ such that the following holds:

$$
\int_{0}^{L} \mathrm{e}^{-\mu x} \overline{\boldsymbol{\xi}}(x)^{\prime} P_{2} \overline{\boldsymbol{\xi}}(x) d x \leq \int_{0}^{L} \overline{\boldsymbol{\xi}}(x)^{\prime} P_{2} \overline{\boldsymbol{\xi}}(x) d x \leq \varrho \int_{0}^{L} \overline{\boldsymbol{\xi}}(x)^{\prime} \overline{\boldsymbol{\xi}}(x) d x \leq \varrho \sigma .
$$

In the above inequality, observe that $\varrho$ is any scalar equal or larger than the largest eigenvalue of $P_{2}$.

Then, the following upper bound on $V(\overline{\boldsymbol{\xi}})$ is obtained

$$
V(\overline{\boldsymbol{\xi}}) \leq 1+\varrho \sigma
$$

as soon as $\overline{\boldsymbol{\xi}} \in \mathcal{D}_{1}, \overline{\boldsymbol{\xi}}_{\text {in }} \in \mathcal{D}_{2}$ which, in view of the above, implies that:

$$
V(\boldsymbol{\xi}(t, \cdot)) \leq \gamma, \gamma=1+\varrho \sigma, \forall t \in \mathbb{R}^{+}, \delta \in \Delta .
$$

Now, let $V_{1}\left(\boldsymbol{\xi}_{\text {in }}\right)=\boldsymbol{\xi}_{\text {in }}^{\prime} P_{1} \boldsymbol{\xi}_{\text {in }}$, then it follows from (10) that:

$$
V_{1}\left(\boldsymbol{\xi}_{\text {in }}(t)\right) \leq V(\boldsymbol{\xi}(t, \cdot)) \leq \gamma, \forall t \in \mathbb{R}^{+}, \delta \in \Delta .
$$

Let the following set:

$$
\mathcal{R}_{1}:=\left\{\boldsymbol{\xi}_{\text {in }}: V_{1}\left(\boldsymbol{\xi}_{\text {in }}\right)=\boldsymbol{\xi}_{\text {in }}^{\prime} P_{1} \boldsymbol{\xi}_{\text {in }} \leq \gamma\right\} .
$$

Thus, the condition in (16) guarantees that $\boldsymbol{\xi}_{\text {in }} \in \mathcal{R}_{1}$ for all $t \in \mathbb{R}^{+}$and $\delta \in \Delta$, since $V_{1}\left(\boldsymbol{\xi}_{\text {in }}(t)\right) \leq V(\boldsymbol{\xi}(t, \cdot)) \leq V(\overline{\boldsymbol{\xi}}) \leq \gamma$ for all $t \in \mathbb{R}^{+}$and $\delta \in \Delta$. Then, provided that $\mathcal{R}_{1} \subset \Xi$, the condition in (12) holds along the solutions to (1) and (2) for all $\overline{\boldsymbol{\xi}} \in \mathcal{D}_{1}$ and $\overline{\boldsymbol{\xi}}_{\text {in }} \in \mathcal{D}_{2}$.

In the sequel, it is introduced the main result of this section which proposes a numerical and tractable solution to problem $\boldsymbol{P} \mathbf{1}$ in terms of a finite 
set of LMI constraints. To this end, it is assumed that $\Xi$ is a given symmetric hyper-rectangle (i.e., a polytopic region) containing $\boldsymbol{\xi}_{\text {in }}=0$ with known vertices. Moreover, $\Xi$ can be also equivalently defined in terms of its faces as below

$$
\Xi=\left\{\boldsymbol{\xi}_{\text {in }} \in \mathbb{R}^{n}:\left|c_{i}^{\prime} \boldsymbol{\xi}_{\text {in }}\right| \leq 1, i=1, \ldots, n_{f}\right\},
$$

with $c_{i} \in \mathbb{R}^{n}, i=1, \ldots, n_{f}$, defining the faces of $\Xi$.

Theorem 1 (Stability Analysis). Consider the PDE-ODE system (1) and (2), with the initial conditions defined by (7) and (8), and given control gains $G\left(\boldsymbol{\xi}_{\text {in }}\right)$ and $K$. Let $\Xi$ and $\Delta$ be given polytopes, and $\sigma, \mu$ be given positive scalars. Suppose there exist diagonal matrices $P_{1}$ and $P_{2}$, a matrix $W$, with appropriate dimensions, and a positive scalar @ satisfying the following:

$$
\begin{aligned}
& P_{1}>0, P_{2}>0, \varrho \boldsymbol{I}_{n}-P_{2} \geq 0, \\
& {\left[\begin{array}{cc}
\gamma & \gamma c_{j}^{\prime} \\
\gamma c_{j} & P_{1}
\end{array}\right] \geq 0, j=1, \ldots, n_{f},} \\
& \Theta\left(\boldsymbol{\xi}_{i n}, \delta, \mu\right)+\operatorname{He}\left\{W \mathcal{M}\left(\boldsymbol{\xi}_{i n}\right)\right\}<0, \\
& \forall\left(\boldsymbol{\xi}_{\text {in }}, \delta\right) \in \mathcal{V}(\Xi \times \Delta),
\end{aligned}
$$

where $\gamma=1+\varrho \sigma$ and

$$
\begin{aligned}
& \Theta\left(\boldsymbol{\xi}_{\text {in }}, \delta, \mu\right)=\left[\begin{array}{c|c}
N^{\prime}\left(A\left(\boldsymbol{\xi}_{i n}, \delta\right)^{\prime} P_{1}+P_{1} A\left(\boldsymbol{\xi}_{i n}, \delta\right)+\boldsymbol{\Lambda}(\delta) P_{2}+\mu \boldsymbol{\Lambda}(\delta) P_{1}\right) N & N^{\prime} P_{1} B\left(\boldsymbol{\xi}_{i n}, \delta\right) K \\
+\mathcal{G}^{\prime} B\left(\boldsymbol{\xi}_{\text {in }}, \delta\right)^{\prime} P_{1} N+N^{\prime} P_{1} B\left(\boldsymbol{\xi}_{\text {in }}, \delta\right) \mathcal{G} & N^{\prime}\left(-\mathrm{e}^{-\mu} \boldsymbol{\Lambda}(\delta) P_{2}\right) N
\end{array}\right], \\
& N=\left[\begin{array}{ll}
\boldsymbol{I}_{n} & \mathbf{0}_{n \times n^{2}}
\end{array}\right], \mathcal{G}=\left[\begin{array}{llll}
G_{0} & G_{1} & \cdots & G_{n}
\end{array}\right], \\
& \mathcal{M}\left(\xi_{\text {in }}\right)=\left[\begin{array}{cc|c}
\Pi\left(\boldsymbol{\xi}_{\text {in }}\right) & -\boldsymbol{I}_{n^{2}} & \mathbf{0} \\
\mathbf{0} & \mathcal{N}\left(\boldsymbol{\xi}_{\text {in }}\right) & \mathbf{0}
\end{array}\right], \Pi(\xi)=\left[\begin{array}{c}
\xi_{(1)} \otimes \boldsymbol{I}_{n} \\
\vdots \\
\xi_{(n)} \otimes \boldsymbol{I}_{n}
\end{array}\right] \\
& \mathcal{N}(\xi)=\left[\begin{array}{cccccc}
\xi_{(2)} \boldsymbol{I}_{n} & -\xi_{(1)} \boldsymbol{I}_{n} & \mathbf{0} & \ldots & \ldots & \mathbf{0} \\
\mathbf{0} & \xi_{(3)} \boldsymbol{I}_{n} & -\xi_{(2)} \boldsymbol{I}_{n} & \mathbf{0} & \ldots & \mathbf{0} \\
\vdots & \ddots & \ddots & \ddots & \ldots & \vdots \\
\vdots & \ldots & \ddots & \ddots & \ddots & \vdots \\
\mathbf{0} & \ldots & \ldots & \mathbf{0} & \xi_{(n)} \boldsymbol{I}_{n} & -\xi_{(n-1)} \boldsymbol{I}_{n}
\end{array}\right]
\end{aligned}
$$


Then, the origin of the coupled PDE-ODE system of (1) and (2) is locally robustly exponentially stable in the sense of Definition 1. Moreover, for any $\overline{\boldsymbol{\xi}} \in \mathcal{D}_{1}$ and $\overline{\boldsymbol{\xi}}_{\text {in }} \in \mathcal{D}_{2}$, the system trajectories remain bounded to $\mathcal{R}_{1}$ as defined in (17), for all $t \geq 0$, and vanish to zero as the time goes to infinity.

Proof 1. Suppose the conditions of Theorem 1 are satisfied, then from convexity arguments the condition in (21) is also satisfied for all $\left(\boldsymbol{\xi}_{i n}, \delta\right) \in$ $\Xi \times \Delta, \boldsymbol{\xi}_{\text {out }} \in \mathbb{R}^{n}$.

Firstly, let us show that (21) implies that (12) holds. To do that, let

$$
\Psi\left(\boldsymbol{\xi}_{\text {in }}\right)=\left[\begin{array}{cc}
\Pi_{a}\left(\boldsymbol{\xi}_{\text {in }}\right) & \mathbf{0} \\
\mathbf{0} & \boldsymbol{I}_{n}
\end{array}\right], \Pi_{a}(\xi)=\left[\begin{array}{c}
\boldsymbol{I}_{n} \\
\Pi(\xi)
\end{array}\right] .
$$

Hence, pre- and post-multiplying (21) by $\Psi\left(\boldsymbol{\xi}_{\text {in }}\right)^{\prime}$ and $\Psi\left(\boldsymbol{\xi}_{\text {in }}\right)$, respectively, yields

$$
\Phi\left(\boldsymbol{\xi}_{\text {in }}, \delta\right)<0, \forall\left(\boldsymbol{\xi}_{\text {in }}, \delta\right) \in \Xi \times \Delta,
$$

since $\mathcal{M}\left(\boldsymbol{\xi}_{\text {in }}\right) \Psi\left(\boldsymbol{\xi}_{\text {in }}\right)=0$ by construction, and thus

$$
m_{2}\left(\boldsymbol{\xi}_{\text {in }}, \boldsymbol{\xi}_{\text {out }}, \delta\right) \leq 0, \forall\left(\boldsymbol{\xi}_{\text {in }}, \delta\right) \in \Xi \times \Delta, \boldsymbol{\xi}_{\text {out }} \in \mathbb{R}^{n} .
$$

Next, in light of the definition of $V(\boldsymbol{\xi})$ in (10) and taking (11)-(12) into account, the following holds:

$$
\dot{V}(\boldsymbol{\xi}(t, \cdot)) \leq-\mu \epsilon V(\boldsymbol{\xi}(t)), \forall t \in \mathbb{R}^{+} .
$$

Now, notice that (20) implies $\mathcal{R}_{1} \subset \Xi$; see, e.g., [6]. Hence, for all $\overline{\boldsymbol{\xi}} \in \mathcal{D}_{1}$ and $\overline{\boldsymbol{\xi}}_{\text {in }} \in \mathcal{D}_{2}$, it follows that $V_{1}\left(\boldsymbol{\xi}_{\text {in }}(t)\right) \leq V(\boldsymbol{\xi}(t, \cdot)) \leq V(\overline{\boldsymbol{\xi}}) \leq \gamma$ for $t \in \mathbb{R}^{+}$ and $\delta \in \Delta$, which completes the proof.

Theorem 1 can be applied to obtain the largest estimate of the set $\mathcal{D}_{2}$ of admissible initial boundary states assuming that $\mathcal{D}_{1}$ and $\Xi$ are given $a$ priori. For instance, the volume of $\mathcal{D}_{2}$ can be approximately maximized by minimizing the trace of $P_{1}$ (since the trace of $P_{1}^{-1}$ is the sum of the squared semi-axis lengths of $\mathcal{D}_{2}$ ) leading to the following optimization problem

$$
\min _{\mu, Q, P_{1}, P_{2}, W} \text { trace }\left\{P_{1}\right\} \text { subject to }(19)-(21) \text {. }
$$

Notice that the matrix inequalities in (19)-(21) become LMIs when $\mu$ is given. Hence, it can be applied a line search over $\mu$ in order to obtain a solution to optimization problem in (23) via semi-definite programming. 
Remark 2. To be precise, the Lyapunov function in (10) is not differentiable along all $L^{2}$ solutions. However it is continuously differentiable along the smooth solutions, and thus, by density, the analysis developed in this section is also correct for $L^{2}$ solutions. See, for instance, [5, page 67] for further details.

\section{Regional Stabilization}

This section addresses the problem of designing the state feedback gain matrices $G(\cdot)$ and $K(\cdot)$ such that the coupled PDE-ODE system of (1) and (2) with (7) is regionally exponentially stable for all $\delta \in \Delta$. The proposed design will be based on Theorem 1 together with a similarity transformation and matrix parameterizations involving the Lyapunov matrix $P_{1}$.

Thus, consider the following definitions:

$$
\begin{gathered}
\boldsymbol{\eta}_{\text {in }}=P_{1} \boldsymbol{\xi}_{\text {in }}, \boldsymbol{\eta}_{\text {out }}=P_{1} \boldsymbol{\xi}_{\text {out }}, \boldsymbol{\eta}=P_{1} \boldsymbol{\xi}, Q_{1}=P_{1}^{-1}, \\
Q_{2}=Q_{1} P_{2} Q_{1}, G_{p}\left(\boldsymbol{\xi}_{\text {in }}\right)=G\left(\boldsymbol{\xi}_{\text {in }}\right) Q_{1}, K_{p}=K Q_{1}
\end{gathered}
$$

with $Q_{1}>0$.

Now, observe that $m_{1}(\boldsymbol{\xi}, \delta)$ and $m_{2}\left(\boldsymbol{\xi}_{\text {in }}, \boldsymbol{\xi}_{\text {out }}, \delta\right)$ in (11) can be respectively recast as follows:

$$
\begin{gathered}
m_{1}(\boldsymbol{\xi}, \delta)=\mu\left(\boldsymbol{\eta}_{\text {in }}^{\prime} Q_{1} \boldsymbol{\Lambda}(\delta) \boldsymbol{\eta}_{\text {in }}+\int_{0}^{L} \mathrm{e}^{-\mu x} \boldsymbol{\eta}(t, x)^{\prime} Q_{2} \boldsymbol{\Lambda}(\delta) \boldsymbol{\eta}(t, x) d x\right), \\
m_{2}\left(\boldsymbol{\xi}_{\text {in }}, \boldsymbol{\xi}_{\text {out }}, \delta\right)=\boldsymbol{\eta}_{a}^{\prime} \Phi_{p}\left(\boldsymbol{\xi}_{\text {in }}, \delta\right) \boldsymbol{\eta}_{a}
\end{gathered}
$$

where

$$
\begin{gathered}
\boldsymbol{\eta}_{a}=\left[\begin{array}{c}
\boldsymbol{\eta}_{\text {in }} \\
\boldsymbol{\eta}_{\text {out }}
\end{array}\right], \\
\Phi_{p}\left(\boldsymbol{\xi}_{\text {in }}, \delta\right)=\left[\begin{array}{c|c}
Q_{1} A\left(\boldsymbol{\xi}_{\text {in }}, \delta\right)^{\prime}+A\left(\boldsymbol{\xi}_{\text {in }}, \delta\right) Q_{1}+ & \\
G_{p}\left(\boldsymbol{\xi}_{\text {in }}\right)^{\prime} B\left(\boldsymbol{\xi}_{\text {in }}\right)^{\prime}+B\left(\boldsymbol{\xi}_{\text {in }}\right) G_{p}\left(\boldsymbol{\xi}_{\text {in }}\right) & B\left(\boldsymbol{\xi}_{\text {in }}, \delta\right) K_{p} \\
+Q_{2} \boldsymbol{\Lambda}(\delta)+\mu Q_{1} \boldsymbol{\Lambda}(\delta) & \\
\hline K_{p}^{\prime} B\left(\boldsymbol{\xi}_{\text {in }}, \delta\right)^{\prime} & -\mathrm{e}^{-\mu} \boldsymbol{\Lambda}(\delta) Q_{2}
\end{array}\right] .
\end{gathered}
$$

Hence, using similar arguments to Section 3, the condition $\dot{V}(\boldsymbol{\xi}(t, \cdot)) \leq$ $-\mu \epsilon V(\boldsymbol{\xi}(t, \cdot))$ will hold for $t \in \mathbb{R}^{+}$if $m_{2}\left(\boldsymbol{\xi}_{\text {in }}, \boldsymbol{\xi}_{\text {out }}, \delta\right)$ as defined in (26) satisfies

$$
m_{2}\left(\boldsymbol{\xi}_{\text {in }}, \boldsymbol{\xi}_{\text {out }}, \delta\right) \leq 0, \forall\left(\boldsymbol{\xi}_{\text {in }}, \delta\right) \in \Xi \times \Delta, \boldsymbol{\xi}_{\text {out }} \in \mathbb{R}^{n} .
$$


In addition, notice that the condition $\varrho \boldsymbol{I}_{n}-P_{2} \geq 0$ was considered in Section 3 to obtain a bound $\gamma$ on $V(\boldsymbol{\xi}(t, \cdot))$ such that (16) holds for all $t \in \mathbb{R}^{+}$and $\delta \in \Delta$. To this end, the following result will be instrumental to obtain a numerical and tractable solution.

Lemma 1 ([20]). Let $P$ and $R$ be real square matrices with $P>0$ and $R$ nonsingular. Then, the following holds:

$$
R^{\prime} P^{-1} R \geq R+R^{\prime}-P
$$

Because of the parametrization of $P_{2}$ in (24), it follows that $\varrho \boldsymbol{I}_{n}-P_{2} \geq 0$ can be equivalently written as follows:

$$
Q_{1} Q_{2}^{-1} Q_{1}-\varphi \boldsymbol{I}_{n} \geq 0
$$

with $\varphi=\varrho^{-1}$, and noting that $P_{2}=Q_{1}^{-1} Q_{2} Q_{1}^{-1}$.

Hence, the following inequality is a sufficient condition for (29) to hold:

$$
2 Q_{1}-Q_{2}-\varphi \boldsymbol{I}_{n} \geq 0
$$

by applying Lemma 1 with $R=Q_{1}$ and $P=Q_{2}$.

In view of the above developments, it is proposed in the sequel an LMIbased result for designing the state feedback gain matrices $G\left(\boldsymbol{\xi}_{\text {in }}\right)$ and $K$ such that the origin of the coupled PDE-ODE of (1) and (2) is robustly regionally stable in closed-loop.

Theorem 2 (Regional Stabilization). Consider the PDE-ODE system (1) and (2), with the initial conditions defined by (7) and (8). Let $\Xi$ and $\Delta$ be given polytopes, and $\varphi, \sigma$ and $\mu$ be given positive scalars. Suppose there exist diagonal matrices $Q_{1}$ and $Q_{2}$, and matrices $W, S, F_{i}, i=0,1, \ldots, n$, with appropriate dimensions, satisfying the following:

$$
\begin{aligned}
& Q_{1}>0, Q_{2}>0,2 Q_{1}-Q_{2}-\varphi \boldsymbol{I}_{n} \geq 0, \\
& 1-\gamma c_{j}^{\prime} Q_{1} c_{j} \geq 0, j=1, \ldots, n_{f}, \\
& \Theta_{p}\left(\boldsymbol{\xi}_{\text {in }}, \delta, \mu\right)+\operatorname{He}\left\{W \mathcal{M}\left(\boldsymbol{\xi}_{i n}\right)\right\}<0, \\
& \forall\left(\boldsymbol{\xi}_{i n}, \delta\right) \in \mathcal{V}(\Xi \times \Delta),
\end{aligned}
$$


where $\gamma=1+\sigma \varphi^{-1}$ and

$$
\begin{aligned}
& \Theta_{p}\left(\boldsymbol{\xi}_{i n}, \delta, \mu\right)=\left[\begin{array}{c|c}
N^{\prime}\left(Q_{1} A\left(\boldsymbol{\xi}_{i n}, \delta\right)^{\prime}+A\left(\boldsymbol{\xi}_{\text {in }}, \delta\right) Q_{1}\right)+\mathcal{F}^{\prime} B\left(\boldsymbol{\xi}_{\text {in }}, \delta\right)^{\prime} N & N^{\prime} B\left(\boldsymbol{\xi}_{i n}, \delta\right) S \\
+N^{\prime} B\left(\boldsymbol{\xi}_{i n}, \delta\right) \mathcal{F}+N^{\prime}\left(\boldsymbol{\Lambda}(\delta) Q_{2}+\mu \boldsymbol{\Lambda}(\delta) Q_{1}\right) N & -\mathrm{e}^{-\mu} \boldsymbol{\Lambda}(\delta) Q_{2}
\end{array}\right], \\
& \mathcal{F}=\left[\begin{array}{llll}
F_{0} & F_{1} & \cdots & F_{n}
\end{array}\right] .
\end{aligned}
$$

Then, the origin of the coupled PDE-ODE system (1) and (2), with

$$
G\left(\boldsymbol{\xi}_{\text {in }}\right)=F_{0} Q_{1}^{-1}+\sum_{i=1}^{n} \boldsymbol{\xi}_{\text {in }(i)} F_{i} Q_{1}^{-1}, \quad K=S Q_{1}^{-1},
$$

is locally robustly exponentially stable in the sense of Definition 1. Moreover, for any $\overline{\boldsymbol{\xi}} \in \mathcal{D}_{1}$ and $\overline{\boldsymbol{\xi}}_{\text {in }} \in \mathcal{D}_{2}$, the solution to the coupled PDE-ODE system remains bounded to $\mathcal{R}_{1}$ as defined in (17), with $P_{1}=Q_{1}^{-1}$ and $P_{2}=Q_{1}^{-1} Q_{2} Q_{1}^{-1}$, for all $t \geq 0$, and vanish to zero as the time goes to infinity.

Proof 2. Suppose the conditions of Theorem 2 are satisfied, then from convexity arguments the condition in (33) is also satisfied for all $\left(\boldsymbol{\xi}_{i n}, \delta\right) \in$ $\Xi \times \Delta, \boldsymbol{\xi}_{\text {out }} \in \mathbb{R}^{n}$.

Firstly, let us show that (33) implies that (27) holds. To do that, let

$$
\Psi\left(\boldsymbol{\xi}_{\text {in }}\right)=\left[\begin{array}{cc}
\Pi_{a}\left(\boldsymbol{\xi}_{\text {in }}\right) & \mathbf{0} \\
\mathbf{0} & \boldsymbol{I}_{n}
\end{array}\right], \Pi_{a}(\xi)=\left[\begin{array}{c}
\boldsymbol{I}_{n} \\
\Pi(\xi)
\end{array}\right] .
$$

Hence, pre- and post-multiplying (33) by $\Psi\left(\boldsymbol{\xi}_{\text {in }}\right)^{\prime}$ and $\Psi\left(\boldsymbol{\xi}_{\text {in }}\right)$, respectively, yields

$$
\Phi_{p}\left(\boldsymbol{\xi}_{\text {in }}, \delta, \mu\right)<0, \forall\left(\boldsymbol{\xi}_{\text {in }}, \delta\right) \in \Xi \times \Delta,
$$

since $\mathcal{M}\left(\boldsymbol{\xi}_{\text {in }}\right) \Psi\left(\boldsymbol{\xi}_{\text {in }}\right)=0$ by construction.

Now, pre- and post multiplying the above by $\boldsymbol{\eta}_{a}^{\prime}$ and $\boldsymbol{\eta}_{a}$, respectively, leads to (12) taking the definitions of $\boldsymbol{\eta}_{\text {in }}$ and $\boldsymbol{\eta}_{\text {out }}$ in (24) into account and the fact that:

$$
G\left(\boldsymbol{\xi}_{\text {in }}\right)=\mathcal{F} \Pi_{a}\left(\boldsymbol{\xi}_{\text {in }}\right) P_{1}, \quad K=S P_{1} .
$$

Next, notice that (32) implies $\mathcal{D} \in \Xi$; see, e.g., [6]. Then, consider the condition $2 Q_{1}-Q_{2}-\varphi \boldsymbol{I}_{n} \geq 0$ on the right-hand side of (31). From Lemma 1, it follows that:

$$
P_{2}^{-1}-\varphi \boldsymbol{I}_{n} \geq 0,
$$


since $P_{2}=Q_{1}^{-1} Q_{2} Q_{1}^{-1}$. Hence, multiplying the above by $P_{2} \varrho$ with $\varrho=\varphi^{-1}$, the following is obtained

$$
\varrho \boldsymbol{I}_{n}-P_{2} \geq 0 .
$$

Thus, the rest of this proof follows straightforwardly from Theorem 1.

Similarly to the stability analysis counterpart, Theorem 2 can be applied for designing the state feedback gain matrices $G\left(\boldsymbol{\xi}_{\text {in }}\right)$ and $K$, as defined in (5), in order to robustly regionally stabilize the coupled PDE-ODE system of (1) and (2) in closed-loop while maximizing the volume of $\mathcal{D}_{2}$ for a given set $\mathcal{D}_{1}$. Thus, the following optimization problem solves problem $\boldsymbol{P} 2$ :

$$
\max _{\mu, \varphi, Q_{1}, Q_{2}, F_{0}, \ldots, S, W} \log \operatorname{det}\left(Q_{1}\right) \text { subject to (31)-(33). }
$$

Notice that the matrix inequalities in (31)-(33) are LMIs when $\varphi$ and $\mu$ are given a priori. Hence, a solution to the above optimization problem is obtained via semi-definite programming by applying a gridding technique over $\varphi$ and $\mu$.

\section{Illustrative Examples}

In this section, two application-based examples are presented to illustrate the results derived in the paper. The first one consists on designing a boundary controller to regulate the outlet temperature in a Poiseuille flow system. Then, in the second one, it is performed a local stability analysis of a lossless transmission line connected to a constant power load.

\subsection{Poiseuille Flow System}

The fluid transport system is normally used for industrial applications such as the ventilation system of mining industry [38] and hydraulic networks [22]. To investigate the phenomenon of fluid transport in a Poiseuille flow subject to dynamic boundary conditions, an experimental setup has been designed to test and validate the proposed results.

The experimental setup consists on a heating column encasing a resistor, a tube, two ventilators, a gas speed meter and three distributed temperature sensors [8]. The control objective is tracking the outlet temperature by driving the power dissipated on the heating resistor at different air flow speeds through the tube. Only the outlet temperature and the flow speed will be 
considered as measurements for a closed-loop boundary control strategy and thus $G\left(\xi_{\text {in }}\right)$ is set to be zero. In this work, it is assumed that the output ventilator is off.

The modeling of the experimental setup is done by considering two subsystems: the heating column and the tube. One-dimensional transport model is used to describe the gas density, speed and pressure variations in the tube. For the dynamic boundary conditions, it is considered a zero-dimensional model of control volume approach with heat exchanges coming from the heating resistor in the column. The perfect gases law is used to convert density on temperature.

System description and modeling are presented in [9, 8]. Applying some simplifications (according [9, Section 4]) the system of PDEs reduces to the following convection equation

$$
\frac{\partial \rho(t, x)}{\partial t}+v(t) \frac{\partial \rho(t, x)}{\partial x}=0,
$$

where $v(t)$ is the time-varying convection parameter of (37). The gas speed $v(t)$ in the tube is measured.

For the dynamic boundary condition, considering that the input mass flow rate to be expressed as $\dot{m}_{i n}=v(t) \rho(t, 0) A_{t}$. Thus, the dynamic boundary condition can be expressed as:

$$
\dot{\rho}(t, 0)=-\frac{R \tilde{\gamma} T_{i n} v(t) A_{t}}{p_{i n} V_{0}} \rho(t, 0)^{2}+\frac{\tilde{\gamma} v(t) A_{t}}{V_{0}} \rho(t, 0)-\frac{R}{p_{i n} V_{0} C_{v}} \rho(t, 0) d Q,
$$

where $A_{t}$ is the tube cross section area.

Temperature Boundary Control

The output temperature boundary control is designed for (37) with boundary conditions (38). Let define the density error as:

$$
\xi=\rho-\rho_{\text {ref }},
$$

where $\rho_{\text {ref }}$ is the desired output density. It is easy to show that system (37) with boundary conditions (38) can be expressed as follows:

$$
\frac{\partial \xi(t, x)}{\partial t}+\boldsymbol{\Lambda}(\delta) \frac{\partial \xi(t, x)}{\partial x}=0
$$

with the boundary conditions:

$$
\dot{\xi}_{i n}=-A(\delta) \xi_{i n}{ }^{2}+a(\delta) \xi_{i n}-B \xi_{i n} d Q, \quad \xi_{0}=\xi_{i n},
$$


where $A(\delta)=\frac{R \tilde{\gamma} T_{i n} v(\delta) A_{t}}{p_{i n} V_{0}}, a(\delta)=\frac{\tilde{\gamma} v(\delta) A_{t}}{V_{0}}$ and $B=\frac{R}{p_{i n} V_{0} C_{v}}$

The speed $v(\delta)$ is measured and is considered an uncertain parameter. The temperature inside the heating column is not measurable. Let define the control input using a feedback (partial) and feedforward linearization as:

$$
d Q=F\left(\delta, \rho_{\text {ref }}\right)+K \xi_{\text {out }}, \quad \xi_{\text {out }}=\xi(t, L),
$$

with $F\left(\delta, \rho_{\text {ref }}\right)=C_{v} \tilde{\gamma} v(\delta) A_{t}\left(\frac{p_{\text {in }}}{R}-T_{\text {in }} \rho_{\text {ref }}\right)$ and $K$ to be designed.

This yields to the system (40) with boundary condition (41), which corresponds to the system considered in Theorem 1 and Theorem 2 . Let define the convex set $\Delta=[1,3]$ as the flow speed $1 \mathrm{~m} / \mathrm{s}$ and $3 \mathrm{~m} / \mathrm{s}$ in order to operate the experimental setup between the temperatures of $300 \mathrm{~K}$ and $325 \mathrm{~K}$.

In order to design the boundary control for system (40) with boundary condition (41), consider the control architecture given in Figure 1.

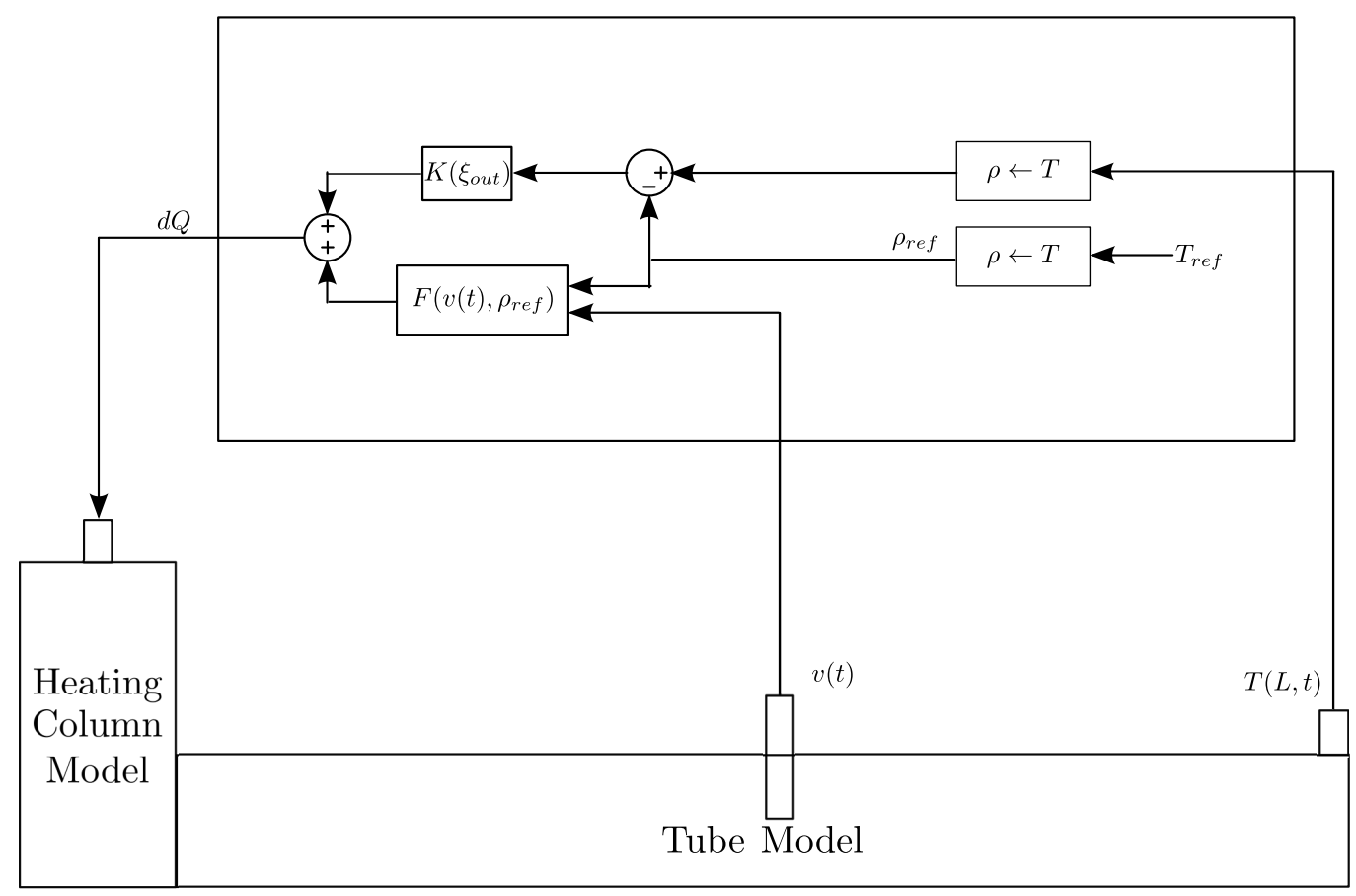

Figure 1: Flow tube control architecture.

In addition, let the following system parameters: adiabatic constant $\tilde{\gamma}=1.4$; molar mass of dry air $M=28.97 \mathrm{~g} / \mathrm{mol} . \mathrm{K}$; ideal gas constant 
$R=8.3143 \mathrm{~J} /(\mathrm{mol} . K)$; specific heat constant for constant pressure $C_{p}=$ $1.005 \mathrm{KJ} / \mathrm{Kg} . \mathrm{K}$ for constant volume $C_{v}=0.718 \mathrm{KJ} / \mathrm{Kg} . \mathrm{K}$; initial pressure $p_{i n}=1$ Bar (or, equivalently, $1 \times 10^{5} \mathrm{~Pa}$ in SI units); initial temperature $T_{\text {in }}=300 \mathrm{~K}$; initial density $\rho=1.1768 \mathrm{Kg} / \mathrm{m}^{3}$ column volume $V_{0}=4 \times 10^{-3} \mathrm{~m}^{3}$; tube cross section area $A_{t}=6.4 \times 10^{-3} \mathrm{~m}^{2}$; and the tube length $L=1 \mathrm{~m}$.

In this scenario, we have applied Theorem 1 with (23) to show that the closed-loop system illustrated in Figure 1 is locally stable considering $K=$ $-0.8, \mu=0.3$ and $\Xi=[-40,40]$. It is displayed in Figure 2 the time response of the outlet temperature's error $e_{L}(t)$ considering a step change of $25 K$ on the outlet temperature's reference. It should be noticed the nice performance achieved by the proposed controller. Nevertheless, the result obtained by the linear controller $u_{L}(t)=-0.8 \boldsymbol{\xi}_{\text {out }}$ can be further improved if a polytopic set $\Xi_{\text {out }}$ bounding the outlet temperature's trajectory $\boldsymbol{\xi}_{\text {out }}(t)$, for all $t \geq 0$, is known a priori which is the case in this example. Then, Theorem 1 with $(23), K\left(\boldsymbol{\xi}_{\text {out }}\right)=-0.8+8 \boldsymbol{\xi}_{\text {out }}, \mu=0.3$ and $\boldsymbol{\xi}_{\text {out }} \in \Xi=[-40,40]$ is applied to demonstrate that the closed loop system is locally stable with the nonlinear control law $u_{N L}=K\left(\boldsymbol{\xi}_{\text {out }}\right) \boldsymbol{\xi}_{\text {out }}$. For illustrative purposes, the closed-loop response of the outlet temperature's error $e_{N L}(t)$ considering the latter control law is also given in Figure 2. It is observed that the convergence of the temperature error with the non-linear gain outperformed the linear counterpart.

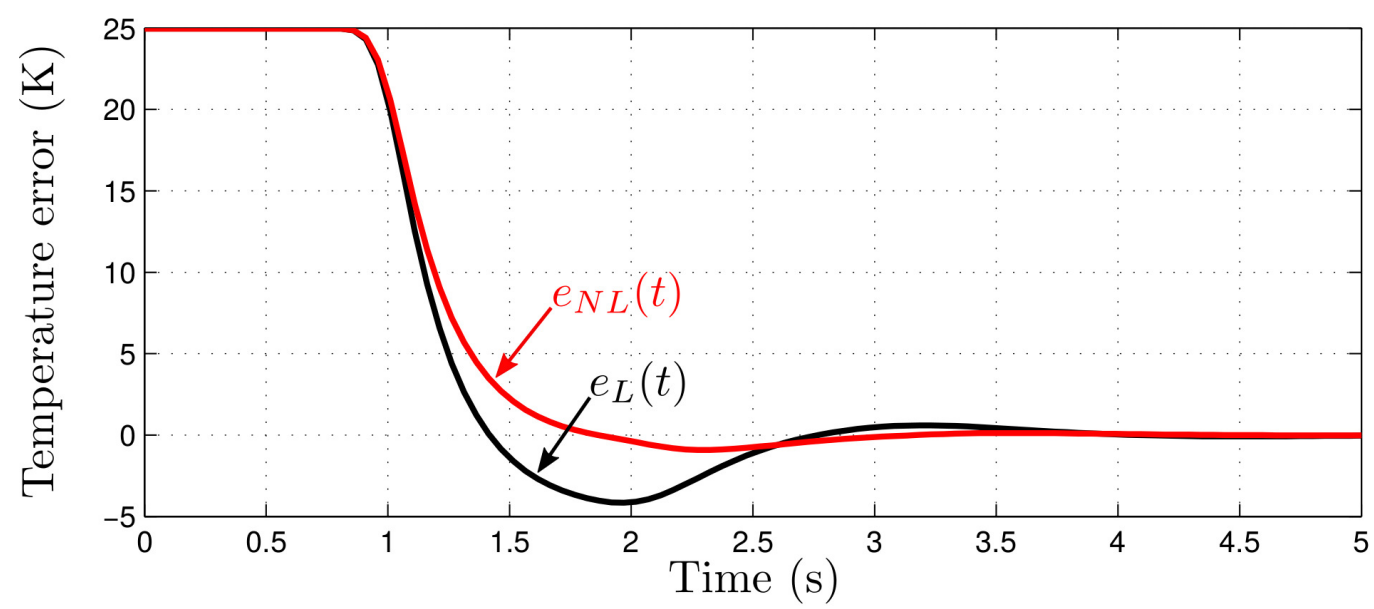

Figure 2: Temperature error trajectories $e_{L}(t)$ and $e_{N L}(t)$ for flow speed $1 \mathrm{~m} / \mathrm{s}$.

Now, in order to illustrate the effectiveness of the proposed nonlinear 
boundary control design strategy, consider system (40) with boundary conditions as defined in (41). Thus, applying Theorem 2 with (36) and considering $K\left(\boldsymbol{\xi}_{\text {out }}\right)=K_{0}+\boldsymbol{\xi}_{\text {out }} K_{1}, \mu=0.5$ and $\Xi=[-40,40]$ leads to the following control gains:

$$
K_{0}=-0.9, \quad K_{1}=9.5 .
$$

Figure 3 presents the closed-loop simulations for the latter control gains considering a step change on the outlet temperature's reference from $300 \mathrm{~K}$ to $325 K$ and three different flow speeds (namely, $v=1 \mathrm{~m} / \mathrm{s}, v=2 \mathrm{~m} / \mathrm{s}$ and $v=3 \mathrm{~m} / \mathrm{s}$ ). Notice that the outlet temperature's trajectory robustly follows the reference and, as expected, the faster is the flow speed, the faster is the convergence time of the fluid transport. In addition, Figure 4 shows the respective control inputs obtained for the latter simulations.

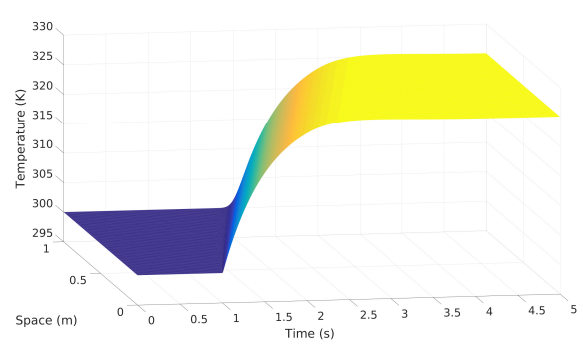

(a) Flow speed $1 \mathrm{~m} / \mathrm{s}$

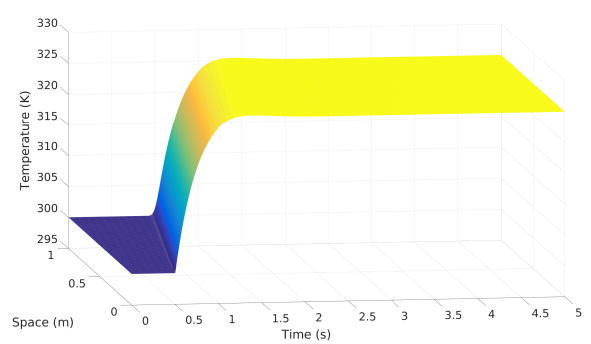

(b) Flow speed $2 \mathrm{~m} / \mathrm{s}$

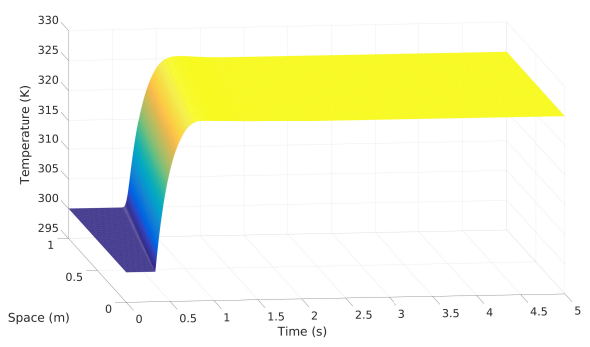

(c) Flow speed $3 \mathrm{~m} / \mathrm{s}$

Figure 3: Output temperature boundary control results using the proposed control (43) to the coupled PDE-ODE experimental setup.

In a scenario similar to that shown in Figure 3, now considering $v(t)=$ $2+\sin (12.56 t)$. Note that in this case, the couple PDE-ODE system is time variant. Without lost of generality, and using gain scheduling, the Theorem 2 is applied with $(36)$ and considering $K\left(\boldsymbol{\xi}_{\text {out }}(t)\right)=K_{0}+\delta(t) \boldsymbol{\xi}_{\text {out }} K_{1}, \mu=0.5$ 


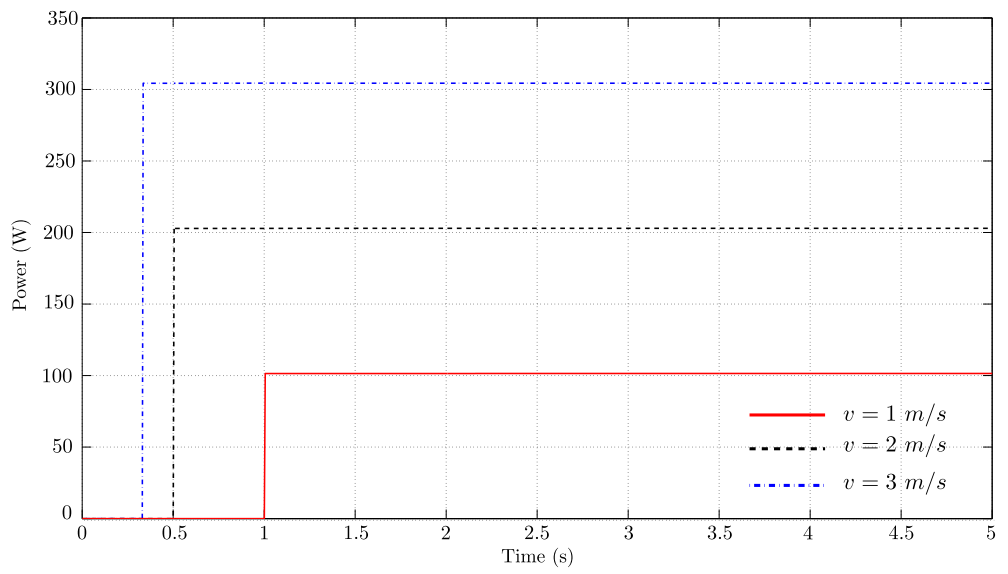

Figure 4: Control inputs for $v=1 \mathrm{~m} / \mathrm{s}, v=2 \mathrm{~m} / \mathrm{s}$ and $v=3 \mathrm{~m} / \mathrm{s}$.

and $\Xi=[-40,40]$ leads to the following control gains:

$$
K_{0}=-0.6 ., \quad K_{1}=7.8 \text {. }
$$

Figure 5 presents the closed-loop simulations for the latter control gains. Notice that the outlet temperature's trajectory robustly follows the reference with a small degradation of transient behavior.

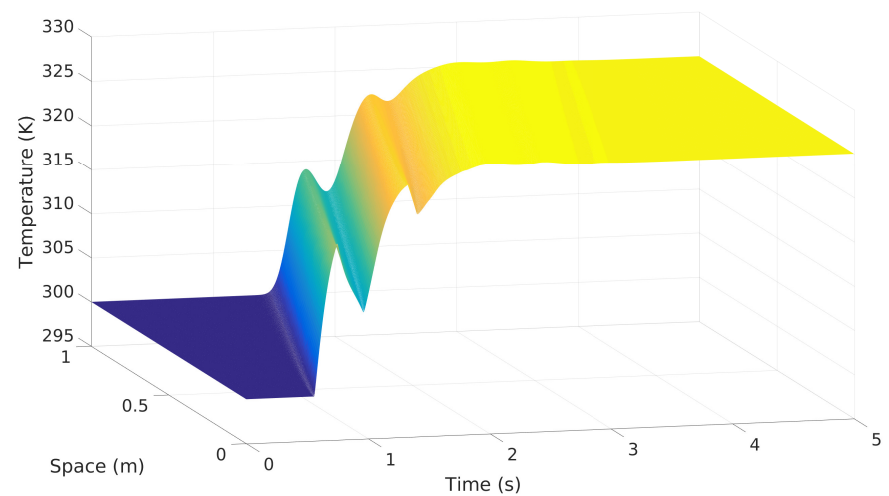

Figure 5: Output temperature boundary control results for $v(t)=2+\sin (12.56 t)$. 


\subsection{Transmission line connected to a Constant Power Load (CPL)}

Consider the lossless electrical line connecting a power supply to a constant power load as shown in Figure 6. The electrical line dynamics (repre-

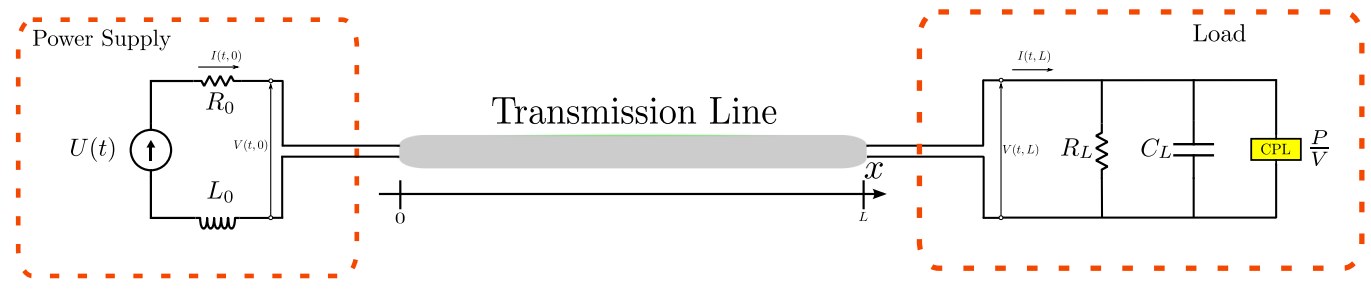

Figure 6: Transmission line connecting an inductive power supply to a Constant Power Load (CPL)

sented by the model of the telegrapher equations [27, page 123], [5, page 18]) is described by the following system of conservation laws:

$$
\begin{aligned}
& \partial_{t} I(t, x)+\frac{1}{L_{\ell}} \partial_{x} V(t, x)=0, \\
& \partial_{t} V(t, x)+\frac{1}{C_{\ell}} \partial_{x} I(t, x)=0,
\end{aligned}
$$

where $I(t, x)$ is the current intensity along the line, $V(t, x)$ is the voltage along the line, $L_{\ell}$ is the line self-inductance per unit length, $C_{\ell}$ is the line capacitance per unit length.

For the circuit represented in Figure 6, the following dynamic boundary conditions are considered:

$$
\begin{gathered}
L_{0} \frac{d I(t, 0)}{d t}+R_{0} I(t, 0)+V(t, 0)=U(t) \\
C_{L} \frac{d V(t, L)}{d t}+\frac{V(t, L)}{R_{L}}-\varphi_{1}\left(V(t, L)-V^{*}\right)+\varphi_{2}\left(V(t, L)-V^{*}\right)^{2}=I(t, L),
\end{gathered}
$$

where $R_{0}$ is the internal resistance of the power supply, and the parallel connection of $R_{L}, C_{L}$ and the CPL represent the load. Notice in the above boundary dynamics that the CPL is approximated by considering the first two terms of a Taylor expansion, with $V^{*}$ being the operation point and

$$
\varphi_{1}=\frac{P}{\left(V^{*}\right)^{2}}, \quad \varphi_{2}=\frac{P}{\left(V^{*}\right)^{3}} .
$$


The operating point is defined by means of a constant input voltage $U^{*}$ leading to the following steady state conditions:

$$
\begin{gathered}
V^{* 2}-\left(\frac{R_{L} U^{*}}{R_{0}+R_{L}}\right) V^{*}+\frac{R_{0} R_{L} P}{R_{0}+R_{L}}=0, U^{* 2}-\left(\frac{4 R_{0} P\left(R_{0}+R_{L}\right)}{R_{L}}\right) \geq 0 \\
I^{*}=\frac{U^{*}-V^{*}}{R_{0}} .
\end{gathered}
$$

Following the modeling technique provided in [5], the Riemann coordinates are defined as follows

$$
\begin{aligned}
& \xi_{1}(t, x) \triangleq\left(V(t, x)-V^{*}\right)+\left(I(t, x)-I^{*}\right) \sqrt{\frac{L_{\ell}}{C_{\ell}}}, \\
& \xi_{2}(t, x) \triangleq\left(V(t, x)-V^{*}\right)-\left(I(t, x)-I^{*}\right) \sqrt{\frac{L_{\ell}}{C_{\ell}}},
\end{aligned}
$$

and thus the inverse coordinates are given by

$$
\begin{aligned}
I(t, x) & =I^{*}+\frac{\xi_{1}(t, x)-\xi_{2}(t, x)}{2} \sqrt{\frac{C_{\ell}}{L_{\ell}}}, \\
V(t, x) & =V^{*}+\frac{\xi_{1}(t, x)+\xi_{2}(t, x)}{2} .
\end{aligned}
$$

Next, expressing the dynamics of (45) and considering Assumption 1 (see Remark 1) and the boundary conditions (46) in Riemann coordinates yields the following system of first-order Hyperbolic equations:

$$
\begin{gathered}
\partial_{t} \boldsymbol{\xi}(t, x)+\boldsymbol{\Lambda} \partial_{x} \boldsymbol{\xi}(t, x)=0, \quad \boldsymbol{\xi}(t, x)=\left[\begin{array}{l}
\xi_{1}(t, x) \\
\xi_{2}^{\prime}(t, x)
\end{array}\right], \\
\xi_{2}^{\prime}(t, L-x)=\xi_{2}(t, x), \\
\boldsymbol{\Lambda}=\left[\begin{array}{cc}
\lambda_{1}+\delta & 0 \\
0 & \lambda_{2}+\delta
\end{array}\right], \lambda_{1}=\lambda_{2} \triangleq \frac{1}{\sqrt{L_{\ell} C_{\ell}}}, \\
{\left[\begin{array}{c}
\dot{X}_{1}(t) \\
\dot{X}_{2}(t)
\end{array}\right]=\left[\begin{array}{cc}
-\alpha_{1}-\delta & 0 \\
0 & -\alpha_{2}-\zeta_{2} X_{2}
\end{array}\right]\left[\begin{array}{l}
X_{1}(t) \\
X_{2}(t)
\end{array}\right]} \\
+\left[\begin{array}{cc}
0 & -\beta_{1} \\
\beta_{2} & 0
\end{array}\right]\left[\begin{array}{c}
\xi_{1}(t, L) \\
\xi_{2}^{\prime}(t, L)
\end{array}\right]+\left[\begin{array}{cc}
0 & \beta_{1}+\delta \\
0 & 0
\end{array}\right] \hat{U}(t) . \\
{\left[\begin{array}{cc}
0 \\
\xi_{1}(t, 0) \\
\xi_{2}^{\prime}(t, 0)
\end{array}\right]=\left[\begin{array}{c}
X_{1}(t) \\
X_{2}(t)
\end{array}\right]+\left[\begin{array}{cc}
0 & 1 \\
-1 & 0
\end{array}\right]\left[\begin{array}{l}
\xi_{1}(t, L) \\
\xi_{2}^{\prime}(t, L)
\end{array}\right],}
\end{gathered}
$$


with

$$
\begin{gathered}
\alpha_{1}=\frac{1}{L_{0}} \sqrt{\frac{L_{\ell}}{C_{\ell}}}+\frac{R_{0}}{L_{0}}, \quad \alpha_{2}=\frac{1}{C_{L}} \sqrt{\frac{C_{\ell}}{L_{\ell}}}+\frac{1}{R_{L} C_{L}}-\frac{\varphi_{1}}{C_{L}}, \\
\beta_{1}=\frac{2}{L_{0}} \sqrt{\frac{L_{\ell}}{C_{\ell}}}, \quad \beta_{2}=\frac{2}{C_{L}} \sqrt{\frac{C_{\ell}}{L_{\ell}}}, \quad \zeta_{2}=\frac{\varphi_{2}}{C_{L}}, \quad \hat{U}(t)=U(t)-U^{*},
\end{gathered}
$$

where the uncertainty $\delta$ has been added to the model to cope with possible modeling errors.

For controlling the output voltage of the circuit represented in Fig. 6, consider the following control law:

$$
\begin{gathered}
\hat{U}(t)=K\left(\boldsymbol{\xi}_{\text {out }}\right) \boldsymbol{\xi}_{\text {out }}, \quad K\left(\boldsymbol{\xi}_{\text {out }}\right)=K_{0}+\sum_{i=1}^{2} \xi_{\text {out }(i)} K_{i}, \quad \boldsymbol{\xi}_{\text {out }}=\left[\begin{array}{l}
\xi_{1}(t, L) \\
\xi_{2}^{\prime}(t, L)
\end{array}\right], \\
K\left(\boldsymbol{\xi}_{\text {out }}\right)=\underbrace{\left[\begin{array}{cc}
0 & k_{1} \\
0 & 0
\end{array}\right]}_{K_{0}}+\xi_{1}(t, L) \underbrace{\left[\begin{array}{cc}
0 & 0 \\
0 & 0
\end{array}\right]}_{K_{1}}+\xi_{2}^{\prime}(t, L) \underbrace{\left[\begin{array}{cc}
0 & k_{2} \\
0 & 0
\end{array}\right]}_{K_{2}}, .
\end{gathered}
$$

with $k_{1}$ and $k_{2}$ being tuning parameters.

To evaluate the local stability of the closed-loop system, it is assumed normalized values of the system parameters, i.e., $R_{0}=1, L_{0}=1, R_{L}=1$, $C_{L}=1, L_{\ell}=1$ and $\left.C_{\ell}\right)=1$. In addition, it is considered that $U^{*}=1$ and the constant output power is set to $P=0.1$ to satisfy the equilibrium conditions given in (47). Also, for this example, the domains of $\boldsymbol{\xi}(t, x)=\overline{\boldsymbol{\xi}}$, $X(t)$ and $\delta$ are supposed to be known a priori.

In order to apply the results given in Section 3, the following domains were considered

$$
\begin{gathered}
\tilde{\mathcal{D}}_{1}:=\left\{\overline{\boldsymbol{\xi}} \in L^{2}\left((0, L) ; \mathbb{R}^{n}\right): \int_{0}^{L} \overline{\boldsymbol{\xi}}(x)^{\prime} \overline{\boldsymbol{\xi}}(x) d x \leq 0.2\right\}, \\
\tilde{\Xi}=\left\{\boldsymbol{X} \in \mathbb{R}^{2}:\left|X_{i}\right| \leq 0.5, i=1,2\right\}, \Delta=\left[\begin{array}{ll}
-0.1 & 0.1
\end{array}\right],
\end{gathered}
$$

leading to the domain of attraction estimate given in Figure 7 obtained from optimization problem (23) with $\mu=0.2$. Notice that the derived estimate

$$
\tilde{\mathcal{D}}_{2}=\left\{\boldsymbol{X} \in \mathbb{R}^{2}: \boldsymbol{X}^{\prime} \tilde{P}_{1} \boldsymbol{X} \leq 1\right\}, P_{1}=\left[\begin{array}{ll}
4.23 & 0.00 \\
0.00 & 4.25
\end{array}\right],
$$


is defined in terms of the auxiliary variable $X(t)$. The projection of the above set in Riemann coordinates can be easily obtained by noticing that $X(t)=M \overline{\boldsymbol{\xi}}(t)$, where

$$
M=\left[\begin{array}{cccc}
1 & 0 & 0 & -1 \\
0 & 1 & 1 & 0
\end{array}\right], \overline{\boldsymbol{\xi}}(t)=\left[\begin{array}{l}
\xi_{1}(t, 0) \\
\xi_{2}^{\prime}(t, 0) \\
\xi_{1}(t, L) \\
\xi_{2}^{\prime}(t, L)
\end{array}\right]
$$

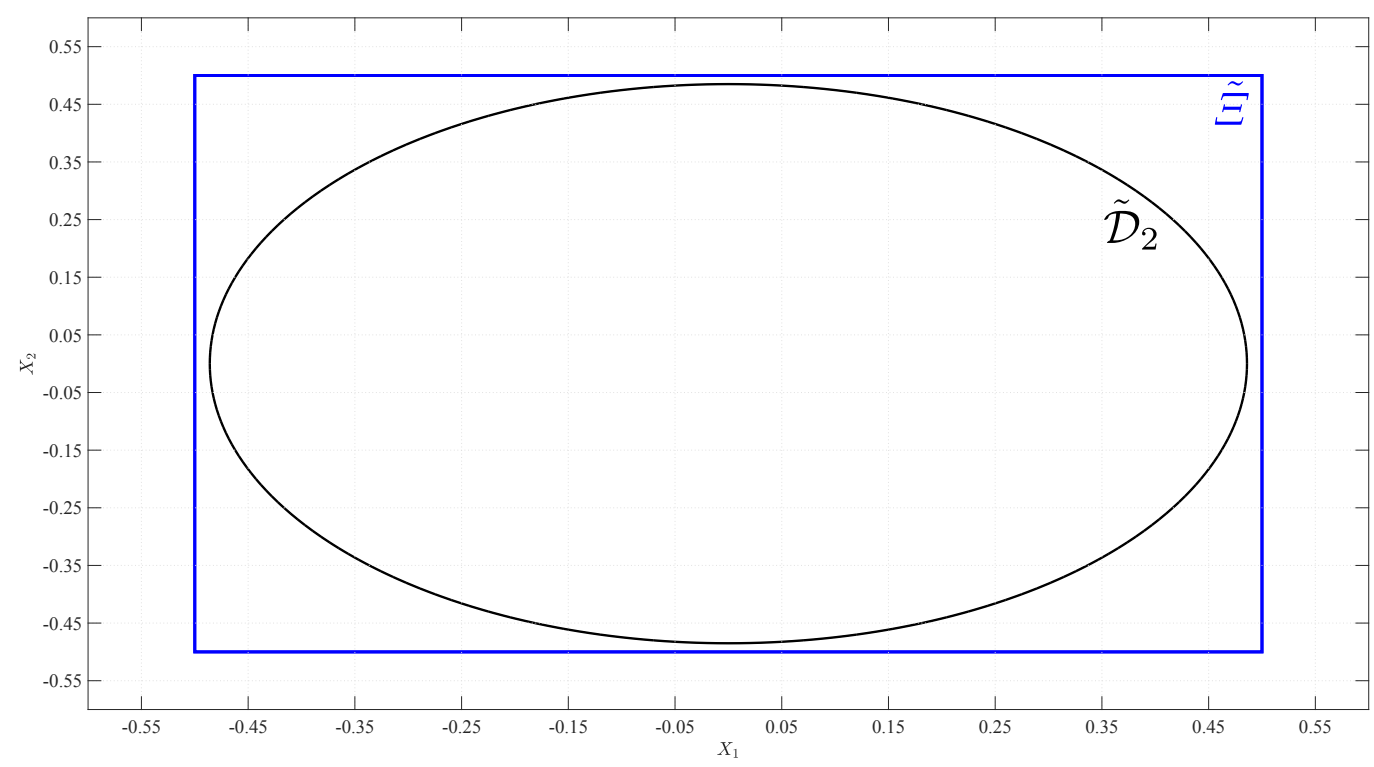

Figure 7: Stability domain $\tilde{\mathcal{D}}_{2}$.

\section{Concluding Remarks}

This paper has focused on the boundary control of uncertain first-order hyperbolic system subject to nonlinear boundary actuation dynamics. An LMI based result has been proposed for assessing the regional robust exponential stability of the closed-loop system. The stability result is then extended to cope with nonlinear boundary control design based on strict Lyapunov functions. Two application-based examples were considered to illustrate the proposed results, where simulation results have shown the effectiveness of the proposed technique. 


\section{References}

[1] Amato, F., Cosentino, C., Fiorillo, A. S., Merola, A., January 2009. Stabilization of bilinear systems via linear state-feedback control. IEEE Transactions on Circuits and Systems II: Express Briefs 56 (1), 76-80.

[2] Amato, F., Cosentino, C., Merola, A., 2007. On the region of attraction of nonlinear quadratic systems. Automatica 43 (12), 2119 - 2123.

[3] Arguello-Serrano, B., Velez-Reyes, M., 1999. Nonlinear control of a Heating, Ventilating, and Air conditioning system with thermal load estimation. IEEE Transactions on Control Systems Technology 7 (1), 56-63.

[4] Bastin, G., Coron, J.-M., 2011. On boundary feedback stabilization of non-uniform linear $2 \times 2$ hyperbolic systems over a bounded interval. Systems \& Control Letters 60 (11), 900-906.

[5] Bastin, G., Coron, J.-M., 2016. Stability and Boundary Stabilization of 1-D Hyperbolic Systems. Birkhauser Basel, Paris, France.

[6] Boyd, S., El Ghaoui, L., Feron, E., Balakrishnan, V., Jun. 1994. Linear Matrix Inequalities in System and Control Theory. Vol. 15 of Studies in Applied Mathematics. SIAM, Philadelphia, PA.

[7] Boyd, S., Vandenberghe, L., 2004. Convex optimization. Cambridge university press.

[8] Caldeira, A. F., Prieur, C., Coutinho, D. F., Leite, V. J. S., September 2015. Modeling and control of flow with dynamical boundary actions. In: 2015 IEEE Conference on Control Applications (CCA). IEEE, Sydney, Australia, pp. 1579-1584.

[9] Castillo, F., Witrant, E., Dugard, L., 2013. Dynamic boundary stabilization of linear parameter varying hyperbolic systems: Application to a Poiseuille flow. IFAC Proceedings Volumes 46 (3), 349-354.

[10] Castillo, F., Witrant, E., Prieur, C., Dugard, L., 2012. Dynamic boundary stabilization of linear and quasi-linear hyperbolic systems. In: Proceedings in $51^{\text {th }}$ IEEE Control and Decision Conference. Maui, Hawaii, pp. 2952-2957. 
[11] Castillo, F., Witrant, E., Prieur, C., Dugard, L., 2013. Boundary observers for linear and quasi-linear hyperbolic systems with application to flow control. Automatica 49 (11), 3180-3188.

[12] Castillo, F., Witrant, E., Prieur, C., Talon, V., Dugard, L., 2015. Fresh air fraction control in engines using dynamic boundary stabilization of LPV hyperbolic systems. IEEE Transactions on Control Systems Technology 23 (3), 963-974.

[13] Chesi, G., 2010. LMI techniques for optimization over polynomials in control: A survey. IEEE Transactions on Automatic Control 55 (11), $2500-2510$.

[14] Christofides, P. D., 2001. Nonlinear and Robust Control of PDE Systems - Methods and Applications to Transport-Reaction Processes. Birkhauser, Boston.

[15] Coron, J.-M., Bastin, G., D’Andréa-Novel, B., 2008. Dissipative boundary conditions for one-dimensional nonlinear hyperbolic systems. SIAM Journal of Control and Optimization 47 (3), 1460-1498.

[16] Coron, J.-M., d'Andréa-Novel, B., Bastin, G., 2007. A Strict Lyapunov Function for Boundary Control of Hyperbolic Systems of Conservation Laws. IEEE Transactions on Automatic Control 52 (1), 2-11.

[17] Coutinho, D. F., De Souza, C. E., 2012. Nonlinear state feedback design with a guaranteed stability domain for locally stabilizable unstable quadratic systems. IEEE Transactions on Circuits and Systems I: Regular Papers 59 (2), 360-370.

[18] Coutinho, D. F., Fu, M., Trofino, A., Danes, P., 2008. $\mathcal{L}_{2}$-gain analysis and control of uncertain nonlinear systems with bounded disturbance inputs. International Journal of Robust and Nonlinear Control 18 (1), 88-110.

[19] Coutinho, D. F., Wouwer, A. V., 2013. A robust non-linear feedback control strategy for a class of bioprocesses. IET Control Theory and Applications 7 (6), 829-841. 
[20] De Oliveira, M. C., Geromel, J. C., Bernussou, J., 2002. Extended $\mathrm{H}_{2}$ and and $\mathrm{H}_{\infty}$ norm characterizations and controller parametrizations for discrete-time systems. International Journal of Control 75 (9), 666-679.

[21] Diagne, A., Bastin, G., Coron, J.-M., 2012. Lyapunov exponential stability of linear hyperbolic systems of balance laws. Automatica 48, 109114 .

[22] Dos Santos, V., Prieur, C., 2008. Boundary control of open channels with numerical and experimental validations. IEEE Transactions on Control Systems Technology 16 (6), 1252-1264.

[23] Dos Santos Martins, V., Rodrigues, M., Diagne, M., 2012. A multimodel approach to Saint-Venant equations: A stability study by LMIs. Applied Mathematics and Computer Science 22 (3), 539-550.

[24] Duan, G.-D., Yu, H.-H., 2013. LMIs in Control Systems. CRC Press, Boca Raton, FL.

[25] Espana, M., Landau, I., 1978. Reduced order bilinear models for distillation columns. Automatica 14 (4), 345-355.

[26] Ge, S. S., He, W., Ee-How, B. V., Choo, Y. S., 2010. Boundary Control of a Coupled Nonlinear Flexible Marine Riser. IEEE Transactions on Control Systms Technology 18 (5), 1080-1091.

[27] Heaviside, O., 1892. Electromagnetic induction and its propagation, $2^{\text {nd }}$ Edition. Vol. 2. In Eletrical Papers, Macmillan and co, London.

[28] Krstic, M., Smyshlyaev, A., 2008. Backstepping boundary control for first-order hyperbolic pdes and application to systems with actuator and sensor delays. Systems \& Control Letters 57 (9), 750-758.

[29] Lamare, P.-O., Girard, A., Prieur, C., 2016. An optimisation approach for stability analysis and controller synthesis of linear hyperbolic systems. ESAIM: Control, Optimisation and Calculus of Variations 22 (4), 1236-1263.

[30] Lofberg, J., 2004. Yalmip: A toolbox for modeling and optimization in matlab. In: Computer Aided Control Systems Design, 2004 IEEE International Symposium on. IEEE, Taipei, Taiwan, pp. 284-289. 
[31] Mohammadpour, J., Scherer, C. W., 2012. Control of Linear Parameter Varying Systems with Applications. Springer, New York.

[32] Papachristodoulou, A., Prajna, S., 2005. A tutorial on sum of squares techniques for systems analysis. In: Proceedings of the American Control Conference. Portland, OR, pp. 2686-2700.

[33] Prieur, C., Girard, A., Witrant, E., 2014. Stability of switched linear hyperbolic systems by lyapunov techniques. IEEE Transactions on Automatic Control 59 (8), 2196-2202.

[34] Tang, Y., Prieur, C., Girard, A., 2015. Tikhonov theorem for linear hyperbolic systems. Automatica 57, 1-10.

[35] Thounthong, P., Pierfederici, S., 2010. A new control law based on the differential flatness principle for multiphase interleaved DC-DC converter. IEEE Transactions on Circuits and Systems II: Express Briefs 57 (11), 903-907.

[36] Valmórbida, G., Tarbouriech, S., Garcia, G., 2010. State feedback design for input-saturating quadratic systems. Automatica 46 (7), 1196 - 1202.

[37] Vidyasagar, M., 2002. Nonlinear Systems Analysis, 2nd Edition. SIAM unabridged republication of original work published by Prentice-Hall.

[38] Witrant, E., Johansson, K., HYNX team, 2008. Air flow modelling in deep wells: aplication to mining ventilation. $4^{\text {th }}$ IEEE Conference on Automation Science and Engineering. 